\title{
Beginning to understand microRNA function
}

\author{
Tingting Du ${ }^{1}$, Phillip D Zamore ${ }^{1}$ \\ ${ }^{1}$ University of Massachusetts Medical School, 364 Plantation Street, Worcester, MA 01605-2324, USA. \\ phillip.zamore@umassmed.edu
}

Cell Research (2007) 17:661-663. doi: 10.1038/cr.2007.67; published online 13 Aug 2007

MicroRNAs (miRNAs) are 22 nt small RNAs expressed by plants, animals, viruses and at least one unicellular organism, the green alga, Chlamydomonas reinhardtii [1]. Most miRNAs are transcribed as primary miRNAs (pri-miRNAs) by RNA polymerase II, although a few are transcribed by RNA polymerase III. In animals, pri-miRNAs are converted to mature miRNAs by two successive endonucleolytic cleavages [2]. The pri-miRNA is first cut in the nucleus by Drosha, a ribonuclease III (RNase III) enzyme, acting with its double-stranded RNA-binding domain (dsRBD) protein partner, called DGCR8 in vertebrates and Pasha in invertebrates, into an $\sim 70 \mathrm{nt}$ stem loop, the precursor miRNA (pre-miRNA). After its export to cytoplasm by Exportin 5, the pre-miRNA is cut into mature miRNA by a second RNase III enzyme, Dicer, which partners in mammals with one of two dsRBD proteins - TRBP (HIV1 tar RNA-binding protein) or PACT, or in Drosophila melanogaster with the dsRBD protein Loquacious (Loqs). The mature miRNA is then loaded into an effector complex, the RNA-induced silencing complex (RISC), whose core component is always a member of the Argonaute (Ago) family of RNA-guided RNA regulatory proteins [3]. Recently, an alternative processing pathway was identified for a distinct sub-group of miRNAs in Drosophila and C. elegans $[4,5]$. These miRNAs exploit the pre-mRNA splicing machinery to generate a pre-miRNA directly, bypassing the processing of a pri-miRNA by Drosha. For these miRNAs, the pre-miRNA is at once the precursor of a mature miRNA and a compact, fully functional intron, hence the name, 'mirtrons'.

In animals, miRNAs typically bind to the 3' untranslated region (UTR) of their target mRNAs through sequences that are only partially complementary. The $5^{\prime}$ region of the miRNA (roughly nucleotides 2-8) contributes disproportionately to target-RNA binding [3]. This 'seed region' is the primary determinant of binding specificity, making
miRNAs surprisingly promiscuous: many miRNAs regulate hundreds of different mRNAs. A common consequence of such seed-mediated miRNA binding is a decrease in the amount of the protein encoded by the target mRNA. However, the precise molecular mechanism of miRNAmediated translational repression remains controversial. In fact, distinct mechanisms of repression have been proposed by different laboratories for different miRNA-target pairs and even for the same miRNA studied with remarkably similar experiments.

Early studies in $C$. elegans suggested that miRNAs blocked protein synthesis after the initiation of translation, because the abundance of repressed mRNA in polyribosomes appeared to be unaltered by miRNA binding [6]. Studies in cultured mammalian cells provided additional support for this model, as a significant fraction of miRNAtarget mRNA remains associated with polyribosomes, despite a large decrease in protein accumulation from these mRNAs [7]. Moreover, the polysomes with which miRNAs associate appear to be translationally active, suggesting that the observed translational inhibition reflects either ribosomes departing the mRNA during protein synthesis or targeted destruction of nascent polypeptide chains as they emerge from the polypeptide exit tunnel $[8,9]$. Yet, other studies in flies and mammals are at odds with these findings, suggesting that miRNAs do, in fact, block mRNA translation at the initiation step [10]. In these experiments, miRNA-directed inhibition requires the 7-methyl guanosine cap, implying a role for miRNA in blocking recognition of the cap by the translation initiation factor eIF4E.

Controversy also dogs the link between miRNA-directed mRNA repression and target mRNA degradation. Some studies report that mRNA levels are unchanged upon miRNA targeting, but others observe destruction of the mRNA upon miRNA binding, perhaps as a consequence of deadenylation and subsequent decapping by standard 
mRNA decay enzymes. Argonaute proteins, miRNAs, and their mRNA targets, all accumulate in cytoplasmic 'Processing bodies' (P-bodies) that function to store and to degrade translationally silenced mRNA [11]. Though there are lines of evidence suggesting a direct role for P-bodies in miRNA-mediated silencing, other data suggest that the movement of miRNA-repressed mRNAs to P-bodies is a consequence, not a cause, of miRNA-directed translational repression [11]. For at least a subgroup of miRNAs, miRNA-mediated translational repression is reversible, with the mRNA shuttling between P-bodies and actively translating polysomes, and the P-body serving as a temporary refuge for miRNA-repressed, translationally quiescent mRNAs [12].

Despite the diversity of modes proposed for miRNAdirected translational inhibition, little is known about the molecular basis of any. Thus, new work by Kiriakidou et al. [14], Chendrimada et al. [15], and Thermann et al. [17] provides long overdue insight into how miRNAs decrease the rate of translational initiation. Remarkably, for the miRNA field, all three studies point to a single explanation for miRNAs' reducing the rate of translational initiation, i.e., the association of ribosomes with the $5^{\prime}$ end of mRNAs.

First, some necessary background. Argonaute proteins lie at the core of all effector complexes containing small RNAs, including miRNAs, small interfering RNAs (siRNAs) and PIWI-interacting RNAs (piRNAs). Argonaute proteins contain a central PAZ domain (named after the family member proteins, Piwi, Argonaute and Zwille), which binds to the $3^{\prime}$ end of single-stranded RNAs, and a carboxy terminal PIWI domain, which binds to the $5^{\prime}$ phosphate of small RNAs [13]. In the June $15^{\text {th }}$ issue of Cell, Kiriakidou and colleagues identify within human Argonaute2 (Ago2) protein a sequence similar to eukaryotic initiation factor 4E (eIF4E) [14]. This motif is present only in the subset of Argonaute proteins implicated in miRNAmediated translational repression, suggesting a special role for this domain in the miRNA pathway. Kiriakidou et al. go on to show that Ago2 binds to a cap-analog resin through the eIF4E-like domain, and that the interaction requires two evolutionarily conservative phenylalanine residues within the domain. Moreover, the cap-binding domain is required for miRNA-directed translational repression at the initiation step, but not when Ago2 regulates mRNA expression by cleaving its mRNA targets, as it does when it mediates siRNA-directed RNA interference (RNAi). The authors propose a straightforward model in which Ago2, bound to the mRNA target by a miRNA, competes with eIF4E for the mRNA cap, reducing the translation of the mRNA target into protein.

Chendrimada and colleagues used a different experi- mental approach to identify a role for a different translation factor, the protein eIF6, in miRNA-directed translational repression [15]. eIF6 has long been known to bind to free $60 \mathrm{~S}$ ribosomal subunits, preventing their joining the $40 \mathrm{~S}$ subunit to generate translationally competent 80 S ribosome particles [16]. Chendrimada et al. purified proteins associated with TRBP, the mammalian homolog of Loquacious, the Drosophila partner of Dicer-1. In mammals, TRBP is thought to participate in both miRNA production and the assembly of miRNAs into Ago2-containing complexes. Among the proteins that co-purified with TRBP were, as expected from earlier work, Dicer and Ago2, but also unexpectedly, protein components of the $60 \mathrm{~S}$ ribosomal subunit and eIF6. Strengthening the case that eIF6 participates in miRNA-mediated mRNA repression, depletion of eIF6 from human cells counteracted miRNA-directed translational repression of a reporter mRNA, and depletion of eIF6 by RNAi in C. elegans decreased the endogenous silencing of mRNAs by miRNAs.

Chendrimada and co-workers propose that miRNAs block translation by recruiting eIF6 to their mRNA targets. eIF6 would then antagonize the joining of the two ribosomal subunits on the miRNA-regulated mRNA. Like Kiriakidou et al. [14], Chendrimada et al. [15] implicate the initiation step of translation as the miRNA regulated step, but unlike the Kiriakidou study, they do not detect a direct role for Argonaute proteins in antagonizing translational initiation. Conceivable, both the model of Kiriakidou et al., which postulates that Ago2 binds the cap, blocking eIF4E binding, and that of Chendrimada et al., which envisions Ago2 as simply increasing the local concentration of eIF6 on the target mRNA, may be true, with the combined action of these two, and perhaps other, translational initiation antagonists explaining miRNA-directed repression.

Contemporaneously, Thermann and colleagues used lysate from Drosophila embryos to recapitulate some aspects of miRNA-directed translational repression for a reporter mRNA bearing in its 3' UTR six copies of an authentic miR-2 binding site [17]. (It is worth noting that the Drosophila mRNA, reaper, from which this miR-2binding site derives, contains only a single copy of the site, not six.) The authors observed a reduction in $80 \mathrm{~S}$ ribosome assembly on the reporter mRNA, suggesting inhibition at the translation initiation step. Surprisingly, some larger messenger ribonucleoprotein particles (mRNPs) formed on the reporter mRNA upon miRNA binding, even when polysome formation was blocked. The authors refer to these particles as 'pseudo-polysomes', because they formed even when $60 \mathrm{~S}$ joining was inhibited, and their formation was insensitive to the polysome disrupting agent puromycin. In theory, these large particles might contain one or both of the ribosomal subunits, but not fully assembled $80 \mathrm{~S}$ 
ribosomes. These data clearly prompt a reevaluation of the polysomes described in early work to be associated with miRNA-regulated mRNAs. The in vitro experiments in flies also support the view that multiple steps in translational initiation, including cap-binding and subunit joining, are regulated by miRNAs: a 7-methyl guanosine cap was required for miRNA-induced silencing in vitro, but the enigmatic 'pseudo-polysomes' still formed when the authentic cap was replaced with a translationally incompetent ApppG cap analog.

While the experiments from these three laboratories add considerable support to the idea that miRNAs repress translational initiation, they do not exclude the possibility that the mechanisms of miRNA-directed mRNA regulation differ among organisms, among miRNAs, or even at different developmental stages. Alternatively, the location of miRNA-binding sites within an mRNA or the position of mismatches and bulges within the miRNA-binding sites may influence the mechanism by which productive translation is repressed. Clearly, much remains to be explained before the molecular basis of miRNA-directed translational repression is clear.

\section{References}

1 Molnar A, Schwach F, Studholme DJ, Thuenemann EC, Baulcombe DC. miRNAs control gene expression in the single-cell alga Chlamydomonas reinhardtii. Nature 2007; 447:1126-1129.

2 Kim VN. MicroRNA biogenesis: coordinated cropping and dicing. Nat Rev Mol Cell Biol 2005; 6:376-385.

3 Zamore PD, Haley B. Ribo-gnome: the big world of small RNAs. Science 2005; 309:1519-1524.

4 Ruby JG, Jan CH, Bartel DP. Intronic microRNA precursors that bypass Drosha processing. Nature 2007; 448:8386.

5 Okamura K, Hagen JW, Duan H, Tyler DM, Lai EC. The Mirtron Pathway Generates microRNA-Class Regulatory RNAs in Drosophila. Cell 2007; 130:89-100.
6 Olsen PH, Ambros V. The lin-4 regulatory RNA controls developmental timing in Caenorhabditis elegans by blocking LIN-14 protein synthesis after the initiation of translation. Dev Biol 1999; 216:671-680.

7 Petersen CP, Bordeleau ME, Pelletier J, Sharp PA. Short RNAs Repress Translation after Initiation in Mammalian Cells. Mol Cell 2006; 21:533-542.

8 Maroney PA, Yu Y, Fisher J, Nilsen TW. Evidence that microRNAs are associated with translating messenger RNAs in human cells. Nat Struct Mol Biol 2006; 13:11021107.

9 Nottrott S, Simard MJ, Richter JD. Human let-7a miRNA blocks protein production on actively translating polyribosomes. Nat Struct Mol Biol 2006; 13:1108-1114.

10 Pillai RS, Bhattacharyya SN, Artus CG, et al. Inhibition of translational initiation by Let-7 MicroRNA in human cells. Science 2005; 309:1573-1576.

11 Eulalio A, Behm-Ansmant I, Izaurralde E. P bodies: at the crossroads of post-transcriptional pathways. Nat Rev Mol Cell Biol 2007; 8:9-22.

12 Bhattacharyya SN, Habermacher R, Martine U, Closs EI, Filipowicz W. Relief of microRNA-mediated translational repression in human cells subjected to stress. Cell 2006; 125:1111-1124.

13 Song JJ, Smith SK, Hannon GJ, Joshua-Tor L. Crystal structure of Argonaute and its implications for RISC slicer activity. Science 2004; 305:1434-1437.

14 Kiriakidou M, Tan GS, Lamprinaki S, De Planell-Saguer M, Nelson PT, Mourelatos Z. An mRNA m(7)G Cap Binding-like Motif within Human Ago2 Represses Translation. Cell 2007; 129:1141-1151.

15 Chendrimada TP, Finn KJ, Ji X, et al. MicroRNA silencing through RISC recruitment of eIF6. Nature 2007; 447:823828.

16 Ceci M, Gaviraghi C, Gorrini C, et al. Release of eIF6 (p27BBP) from the $60 \mathrm{~S}$ subunit allows $80 \mathrm{~S}$ ribosome assembly. Nature 2003; 426:579-584.

17 Thermann R, Hentze MW. Drosophila miR2 induces pseudo-polysomes and inhibits translation initiation. Nature $2007 ; \mathbf{4 4 7}: 875-878$. 